\title{
Planck: Detectability of synchrotron emission by DM annihilation
}

\author{
Eleonora Presani* \\ INAF/OAT - Osservatorio Astronomico di Trieste \\ Loc. Padriciano 410, \\ 34012 Trieste, ITALY \\ E-mail: presani@oats.inaf.it \\ Michele Maris \\ INAF/OAT - Osservatorio Astronomico di Trieste \\ E-mail:maris@oats.inaf.it
}

\section{Anna Gregorio}

Univerity of Trieste- Physics Dep.

E-mail: gregorio@infn.ts.it

\begin{abstract}
In recent years the possibility of indirect detection of WIMPs (Weak Interacting Massive Particles) by observing their final decay or annihilation by-products (gamma-rays, neutrinos, positrons) has been discussed in detail. In particular it has been pointed out as a possible detection method the observation of the synchrotron emission produced by high energy electrons and positrons propagating in the Galactic or in the halo magnetic field. We outline here a short resume of the activity carried out at our site in the framework of the Planck mission. We analyse the conditions in which synchrotron emission from WIMPs annihilation in DM clumps may be detected in Planck. We take into account the DM distribution profile in clumps, the possible range of neutralino masses and cross sections, the annihilation channels and the magnetic field.
\end{abstract}

CMB and Physics of the Early Universe - International Conference

Ischia, Italy

April $20-22,200$

\footnotetext{
${ }^{*}$ Speaker
} 


\section{Introduction}

There is almost universal agreement among astronomers that most of the mass in the Universe and most of the mass in the Galactic halo is dark. Many lines of reasoning suggest that the dark matter consists of some new, as yet undiscovered, particles. SUperSYmmetry theory (SUSY) provides a good candidate: the neutralino $(\chi)$, the lightest supersymmetric stable particle, a linear combination of higgsino, Z-ino and photino states [1]:

$$
\chi^{0}=a \tilde{B}+b \tilde{W}_{3}+c \tilde{H}_{1}^{0}+d \tilde{H}_{2}^{0}
$$

In this equation, $\chi^{0}$ is the neutralino, $\widetilde{B}$ and $\tilde{W}_{3}$ are the supersymmetric partners of the electroweak bosons, $\tilde{H}_{1}^{0}, \tilde{H}_{2}^{0}$ are the neutral higgsinos. The coefficients $a, b, c$ and $d$ indicates the ratio of each component.

A vast experimental effort is focusing to detect WIMPs (Weak Interacting Massive Particle) [2] [3]. A direct detection is difficult as neutralinos interact very weakly with ordinary matter. If neutralinos are Majorana fermions (which means that the particle coincides with its antiparticle), they can self-annihilate producing different combinations of $\mathrm{W}$ ad $\mathrm{Z}$ bosons, mesons, electron pairs and $\gamma$-rays [4] [5]. Several decay channels has been considered, but in most cases the annihilation products adronize in pions which decay in muons that decay in electrons. These electrons are dipped in the Galactic magnetic field, so that they produce synchrotron radiation. Studying and revealing this synchrotron emission may be a unique signature of the presence of neutralinos in our Galaxy.

Indirect searches have been proposed both for $\gamma$-rays and synchrotron emission from the annihilation of WIMPs in the Galactic Center [6][7]. Nevertheless, it is worthy to analyse the possibility of the presence of Dark Matter clumps in the Galactic Halo. In this case we consider the Dark Matter agglomerated in clumps around the halo of the Galaxy. These clumps may be entirely made of Dark Matter or may be identified with some dwarf galaxies near the Milky Way, as the Draco Galaxy [8][9]. Draco galaxy is particulary interesting as is the only known galaxy that shows evidence of a large dark-matter content [10][11].

The reason why we are so interested in the synchrotron emission of WIMPs annihilation is that the predicted fluxes are in the microwave region, and may even exceed the CMB anisotropies signal in some frequencies [6][11][12]. It seems particularly interesting, as we will soon dispone of the new WMAP data and, above all, it will be possible to recognize these clumps with the Planck satellite.

\subsection{Work description}

The clump geometry, its density profile and its position within the Galaxy, as the neutralino physics and the Galactic magnetic field mapping, are parameters of great importance.

In order to develop the model of the synchrotron emission coming from wimps annihilation, we have followed several steps. As main reference, we have used the work of Blasi 
et al.[12]. We studied the whole decay process, both from analytical and Monte Carlo simulation point of view.

In order to understand if it is possible to observe the clump, it is necessary to evaluate the brilliance of synchrotron emission $B_{\downarrow}(d)$, which depends on the frequency $v$ and the impact parameter $d$ with which we see the clump. By definition, the brilliance is the line integral along the line of sight $s$ of the synchrotron emission spectrum $S_{V}(\boldsymbol{R})$ calculated at given point $\boldsymbol{R}$ of the clump. If we choose to express it in units of $\mathrm{GeV} / \mathrm{s} \mathrm{cm}^{3} \mathrm{GHz}$ per solid angle, we have:

$$
B_{v}(d)=\int_{0}^{\infty} S_{v}(\vec{R}(s)) d s
$$

The spectrum $S_{v}(\boldsymbol{R})$ is the integral of the energy spectrum of the electrons emitted by the neutralinos weighted with the single electron:

$$
S_{v}(\vec{R})=\int \frac{d N_{e}}{d E}(\vec{R}, E) \cdot p(v, E) d E
$$

In this equation, $E$ is the electron energy, $v$ is the emission frequency, $d N e / d E$ is the electron spectrum and $p(v, E)$ is the synchrotron emission spectrum [13].

The synchrotron emission spectrum is:

$$
\mathrm{p}(\mathrm{E}, v)=1.165 \times 10^{-5} \mathrm{E}^{2} \mathrm{~B} \sin \alpha \mathrm{F}(\xi) \mathrm{GeV} \mathrm{s}^{-1} \mathrm{GHz}^{-1}
$$

where $F(\xi)$ is derived by a Bessel function and it is calculated numerically.

The total electron spectrum has two main components, derived from two main considerations. The first is the product of the electron spectrum produced by a single neutralino annihilation with the annihilation probability, which is the cross section of the process times the velocity of the neutralinos and the squared number density of the neutralinos. In addition, it is also necessary to take into account another effect. In fact, the presence of a chaotic magnetic field and the Compton diffusion on photons implies that the electrons diffuse in the space [14].

For this reason the spectrum has to be convolved with a Green function, calculated as a solution of the diffusion equation:

$$
\frac{\mathrm{dN}_{\mathrm{e}}}{\mathrm{dE}}=\int d^{3} R \int \mathrm{dE}_{0} \mathrm{G}\left(\mathrm{E}_{0}-\mathrm{E}, \overrightarrow{\mathrm{R}}_{0}-\overrightarrow{\mathrm{R}}\right) \mathrm{W}_{\mathrm{e}}\left(\mathrm{E}_{0}\right) \frac{<\sigma \nu>_{\chi \chi}}{m_{\chi}^{2}} \rho_{\chi}^{2}\left(\vec{R}^{\prime}\right)
$$

where $W_{e}$ is the electrons spectrum for a single annihilation, $E_{0}$ is the electrons initial energy, $\langle\sigma v\rangle_{\chi \chi}$ is the neutralino annihilation cross section (or, better, the expectation value of the product of the cross section with the neutralino velocity) and $\rho_{\chi}(\mathbf{R})$ is the clump density profile.

Beyond the analytical model, which does not provide a good accuracy, in order to calculate the spectrum of the electrons emitted by a single neutralino annihilation, we have used the software package DarkSUSY[15]. This software allows to choose the supersymmetric model by fixing the free parameters of the model. In addition we define the supersymmetric model with all the relative parameters, we have used a particular DarkSUSY routine, which has been kindly provided by prof. Piero Ullio. This code allows to set the annihilation channel. During this work, only two channels have been chosen: the one in fermion and anti-fermion and the one in $W^{+} W$ pairs. These channels are definitely dominant if we consider the neutralino as a pure bino or a pure higgsino. Once the channels have been chosen, the code allows to read the 
numerical tables produced by Pythia, where it is possible to find both the integrated and the differential spectrum of the different final products.

The simulations of the synchrotron emission produced by the neutralino annihilation that are presented in this work have been performed with the software package DarkSYNC, that we have developed, and that includes an interface to use the data obtained with DarkSUSY.

The package includes several routines: a function which computes the synchrotron emission spectrum emitted by the single electron, one that calculates the clump density profile and the relative parameters, one that interpolates the electron spectrum calculated with DarkSUSY in order to obtain the final electron spectrum. Finally, in order to obtain the synchrotron brilliance, the integral along the line of sight is computed. A library which contains many possible clumps has been created, varying all the possible parameters, both with respect to the density profile and the clump distance, and to the physics of the supersymmetric particles (as their mass and the cross section). The main objective of the final part of this work has been to superimpose the three-dimensional model of the clump with the Galactic synchrotron emission maps of the Universe that is today available.

\subsection{Results of the analysis}

Even considering the electron diffusion, the local synchrotron emission distribution is linear in logarithmic scale. It is possible to interpolate it with a straight line for the whole clump extension, until we reach the end of the halo. Beyond this radius the power law changes rapidity and the signal becomes negligible (see Figura 1). This approximation allows to compute in an analytic way the integral along the line of sight, to obtain the brilliance. The signal obtained in

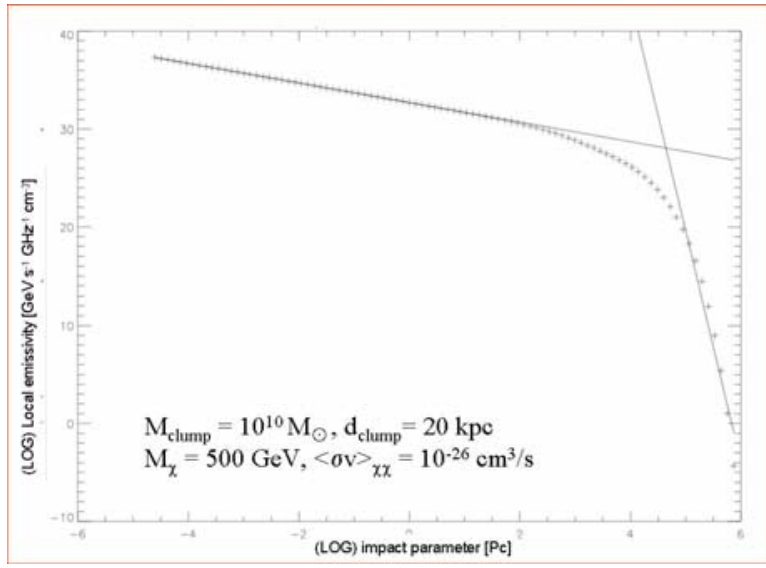
this way has been convoluted with the Planck beam.

Figura 1: Local flux considering the diffusion. We can see that after the end of the clump the signal is negligible.

In order to compare the clump signal with the Galactic synchrotron emission, we used the References Sky Model maps [16]). At this level we decided to compare our result with the synchrotron emission map since in first approximation Planck has the capability to distinguish this signal from all the other diffuse components (CMB, free-free and dust emission).

In order to understand if the clump is detectable, its intensity should be greater than the Galactic synchrotron background fluctuations and the intrinsic background produced by the detectors.

In order to perform this comparison we have implemented a research simulation with the method of open photometry. The open photometry method is aimed at the measure of the total 
observed signal in a small circular area and in a surrounding circular crown, which is used to estimate the background contribution to the central area. The clump is detectable if the noise fluctuations are lower than the signal. We have computed the fluctuations considering both the Galactic diffuse synchrotron emission and the white noise of the detectors. The Galactic fluctuations have been calculated with the open photometry statistics applied to the Reference Sky Model map, while the fluctuations of the white noise have been obtained by considering the Planck scanning strategy and the error propagation theory.

The $44 \mathrm{GHz}$ frequency channel is the most promising, as it seems easier to detect the clump with the chosen method. On the contrary at $30 \mathrm{GHz}$, it is not possible to see the clump. For all the other frequencies the probabilities of revealing the clump are in principle between 70 and $90 \%$, if we do not consider the Galactic centre contribution. These results are very encouraging but we don't take into account the real detection system, as for example the component splitting that Planck should be able to do on the signal.



Figure 2: Map of the Signal/Noise ratio at $44 \mathrm{GHz}$.

It has to be said that the open photometry may give only a basic idea of the clump detectability, as it is based on a constant background, and this hypothesis is not often verified. As it is possible to see in Figure 2, there

are some clear rings with a very different $\mathrm{S} / \mathrm{N}$ ratio all around the map. These rings correspond to local point sources in the Galactic background.

The clumps considered during this work have been treated only from a brilliance point of view. In principle it is not possible to distinguish them from all the other point sources. Several catalogues, as the Reference Sky Model, allows to identify the known point sources so that it will be possible to detect the clump candidate as it will be revealed.

\section{References}

[1] Gerard Jungman, Marc Kamionkowski, Kim Griest - Supersymmetric Dark Matter Phys. Rep. 267 (1996) 195

[2] Bergstrom L., Edsjo J., G. P. 1997, Phys. Rev. D, 55, 1765

[3] Jonathan L. Feng, K. T. M. \& Wilczek, F. 2001, Phys. Rev. D, 63

[4] Ambrosanio, S. \& Mele, B. 1996, Phys. Rev. D, 53, 25412562

[5] Takeshi Nihei, Leszek Roszkowski, R. R. d. A. 2002, Journal of High Energy Physics

[6] Finkbeiner, D. P. 2005, astro-ph/0409027

[7] Cesarini A., Fucito F., L. A. a. 2004, Astroparticle Physics 
[8] Craig, T. 2002, Phys. Rev. D, 66

[9] Tasitsiomi A., Gaskins J., O. A. 2004, Astroparticle Physics

[10] Profumo, S. \& Kamionkowski, M. 2006, astro-ph/0601249

[11] Bergstrom L., H. D. 2006, hep-ph/0512317

[12]Blasi P., Olinto A.V., T. C. 2003, Astroparticle Physics, 18, 649

[13] Ginznburg and Syrovatskii Cosmic Magnetobremsstrahlung Annual Review, NASA astrophysical data system

[14] E.A.Baltz, L.Wai Phys. Rev. D 70, 023512 (2004).

[15] P.Gondolo, J. Edsjö, P. Ullio, L. Bergström, M. Schelke, E.A. Baltz Dark SUSY: Computing Supersymmetric Dark Matter Properties Numerically

[16] http://www.rssd.esa.int/index.php?project=PLANCK 